\title{
Psicología y gamers profesionales en esports: análisis temático y agenda de investigación d
}

\author{
Alejo García-Naveira ${ }^{\text {a1}}{ }^{(0)}$, Eva León Zarceño ${ }^{b}{ }^{(0)}, \&$ Carlos González \\ García (iD) ${ }^{2}$
}

Colegio Oficial de la Psicología, Madrid, España ${ }^{\text {a }}$ Universidad Miguel Hernández, Elche, España ${ }^{\mathrm{b}}$

\section{RESUMEN}

Los objetivos de este trabajo son el realizar una revisión bibliográfica y establecer temas de estudio sobre Psicología y gamers profesionales en esports, así como indicar posibles líneas de investigación. La búsqueda se realizó en las bases de datos Science Direct, PubMed y PsycINFO. El volumen total fue de 101 documentos, publicados entre los años 2012 y el 13 de junio de 2020, de los que se seleccionaron 24 trabajos representativos. Los estudios analizados giran en torno a 8 temáticas fundamentalmente: entrenamiento psicológico, características de personalidad, procesos de equipo, consecuencias negativas de los esports, carrera professional, beneficios de los esports, habilidades y procesos psicológicos y entrenamiento invisible. Las publicaciones de los trabajos son principalmente de investigación. La Revista de Psicología Aplicada al Deporte y al Ejercicio Físico es la que más ha publicado sobre la temática. En conclusión, una revisión exhaustiva evidencia un interés creciente por la temática de los esports y su relación con el ámbito de la Psicología, así como las futuras líneas de investigación que se abren.

\section{Palabras Claves}

esports; gamer profesional; videojuegos; psicólogo del deporte; rendimiento

\begin{abstract}
The goals of this study are to do a bibliographic review and establish study topics on Psychology and professional gamers in esports, as well as finding out possible researching ways about it. The search was done in Science Direct databases, PubMed and PsycINFO. The total amount was 101 documents published between 2012 and the 13 of June of 2020 . From these documents 24 were selected as representatives. The analyzed studies were mainly based on 8 different subjects: psychological training, personality characteristics, team processes, negative consequences of esports, professional career, benefits of esports, psychological skills and processes and invisible training. The Journal Psychology Applied to Sport and Physical Exercise is one of the main contributors to this subject. In conclusion, an exhaustive review shows a growing interest in the subject of esports and its relationship with the field of Psychology, as well as the future lines of research that have been opened.
\end{abstract}

Keywords

esports; professional gamer; video game; sports psychologist; performance

\footnotetext{
1 Correspondence about this article should be addressed to Alejo García-Naveira: alejogarcianaveira@gmail.com

2 Conflicts of Interest: The authors declare that the research was conducted in the absence of any commercial or financial relationships that could be construed as a potential conflict of interest.
} 
Psychology and Professional Gamers in Esports: Thematic Analysis and Research Agenda

\section{Introducción}

El sector de los esports son una realidad de competición, rendimiento, entretenimiento, social, económica, laboral y de investigación (DEV, 2018; Hamari \& Sjöblom, 2017; Martin-Niedecken \& Schättin'2020; Stanton, 2015). A modo de ejemplo, en el año 2019, la audiencia mundial de los esports fue de 453.8 millones de personas, compuesta por 201.2 millones de seguidores habituales y 252.6 millones de espectadores ocasionales, y, además, generó unos ingresos de 1.1 mil millones de dólares, lo que representa un aumento del $26.7 \%$ respecto al año anterior (Newzoo, 2019).

$\mathrm{Al}$ respecto, es necesario diferenciar dos cuestiones relevantes para una mejor comprensión técnica y científico-profesional: los esports vs. videojuegos y el gamer profesional vs. gamer ocasional (Himmelstein et al., 2017). Por un lado, los esports se definen como el juego competitivo organizado de ciertos videojuegos (Antón, 2019). Este autor indica que no todos los videojuegos se pueden considerar esports, los cuales requieren cumplir una serie de criterios como la estructura competitiva (existencia de competiciones, clubes, equipos y gamers profesionales), mediática (comunidad de aficionados, la cobertura mediática, las retransmisiones y los patrocinadores) y el principio de igualdad competitiva (enfrentamiento en igualdad de condiciones entre los competidores). Los principales esports en los cuales se compite mundialmente a nivel individual o en equipo, son, entre otros: League of Legends, DOTA 2, Counter-Strike: Global Offensive, StarCraft II, FIFA, Overwatch, Heroes of the Storm, NBA2KX, Rocket League y Call of Duty (DEV, 2018; Jang \& Byon, 2020).

Por otro lado, un gamer (jugador de esports) compite de forma individual o por equipos contra otro/s (e.g., liga y copa, organizada por entidades como la Liga Profesional de Videojuegos en España), en función del videojuego, sentados delante de una pantalla de ordenador, móvil o monitor de consola (e.g., Play Station) (Bányai et al., 2019; GarcíaNaveira, 2019; Martin-Niedecken \& Schättin, 2020). Estos autores señalan que el gamer profesional tiene como actividad principal los esports, entrena diariamente (físico, técnico-táctico y psicológico), dedicándole un elevado número de horas y compite en eventos reglados y organizados, recibe una remuneración por ello e integra un equipo, o de forma individual, dentro de un club, mientras que el gamer ocasional juega por ocio y recreación sin esos condicionantes anteriormente citados. 
Más allá del debate técnico y legal sobre si los esports son considerados un deporte, con argumentos a favor (Sánchez \& Remilllard, 2018; Thiel \& John, 2018) o en contra (Chiva-Bartoll et al., 2018; Parry, 2018), queda patente que los esports son una actividad humana de rendimiento, con un alto contenido mental, el cual, a nivel profesional, además de entrenar al videojuego, necesita de una preparación física (e.g., resistencia), técnica-táctica (e.g., ejecución y estrategia) y psicológica (e.g., motivación y concentración), en que el cuidado de la salud cobra una especial atención (e.g., problemas físicos y vulnerabilidad psicológica), al igual que sucede con los deportes tradicionales de competición (Bányai et al., 2018; Campbell et al., 2018; Martin-Niedecken \& Schättin, 2020; ONTIER, 2018). Inclusive, se ha sugerido que los requisitos mentales a los que están sujetos los gamers profesionales son similares a los que necesitan los deportistas tradicionales, como, por ejemplo, la comunicación, el trabajo en equipo, control del estrés, etc. (García \& Chamorro, 2018; García-Naveira \& Cantón, 2020; Himmelstein et al., 2017).

A pesar de que ha habido un crecimiento del sector de los esports en los últimos años, existe un contraste con el desarrollo del conocimiento científico-profesional, ya que el tema es relativamente nuevo para los investigadores y profesionales, por lo que se requiere profundizar sobre la temática (Hulk, 2019; Martin-Niedecken \& Schättin, 2020; Pluss et al., 2019). Y en concreto, los esports pueden considerarse como un tipo diferente de uso de los videojuegos, mientras que los gamers profesionales, suponen una clase específica de jugador de videojuegos muy poco estudiado a nivel psicológico, por lo que existe la necesidad de avanzar en esta área de conocimiento (Bányai et al., 2019; Bányai et al., 2018; Pérez-Rubio et al., 2017).

Por ello, el propósito de este estudio es realizar una revisión bibliográfica y desarrollar un análisis temático sobre Psicología y gamers profesionales en esports. Los análisis temáticos difieren de otros tipos de revisiones (e.g., sistemáticas o meta-análisis) en que el objetivo no es probar hipótesis científicas, sino más bien, informar sobre temas de estudio (Grant \& Booth, 2009). Este planteamiento permite a los investigadores revisar una literatura más amplia, el cual puede ayudar a identificar lagunas o áreas para profundizar, así como ofrecer una agenda de investigación para el progreso de la Psicología en los esports. 


\section{Método}

\section{Criterio de búsqueda y de selección}

La búsqueda de la literatura se realizó en las siguientes bases de datos: ScienceDirect, PubMed y PsycINFO. Se identificaron todos los trabajos científicosprofesionales en las bases de datos mencionadas que cumplieran con los criterios de selección. Se tuvo en cuenta el título y resúmenes de cada uno de ellos, que abarcan el periodo temporal desde el año 2012 hasta el 13 de junio de 2020.

Para realizar una óptima búsqueda de producciones científicas, y así garantizar la validación de las mismas, se han tenido en cuenta diversos criterios partiendo de la declaración PRISMA (Urrútia \& Bonfill, 2010). Para seleccionar e incluir los trabajos revisados en el presente análisis temático, se requería cumplir los siguientes criterios: los términos de la búsqueda incluyeron los descriptores en español e inglés de "profesional gamers", "esports" y términos relacionados con "Psychology". Se utilizó el operador booleano "and" para combinarlos. Las búsquedas incluyeron artículos científicos, en español e inglés, realizados con humanos.

Respecto a los criterios de exclusión, se marcaron los siguientes: artículos cuyo contenido estuviera relacionado con videojuegos, con gamer ocasional, los capítulos, libros y tesis doctorales, trabajos de fin de grado o máster, los resúmenes de conferencias de congresos, artículos que estuvieran duplicados en las bases de datos, trabajos que no se realizaban con humanos (e.g., con ordenadores) y estudios de caso único.

Una vez realizadas las búsquedas que cumplían con los requisitos establecidos para su inclusión $(n=101)$, se aplicaron los criterios exclusión marcados, obteniendo finalmente 24 documentos para su análisis (ver Figura 1). 
Bases de datos electrónicas

PsycINFO, ScienceDirect, PubMed,

$(n=26)+(n=58)+(n=17)$

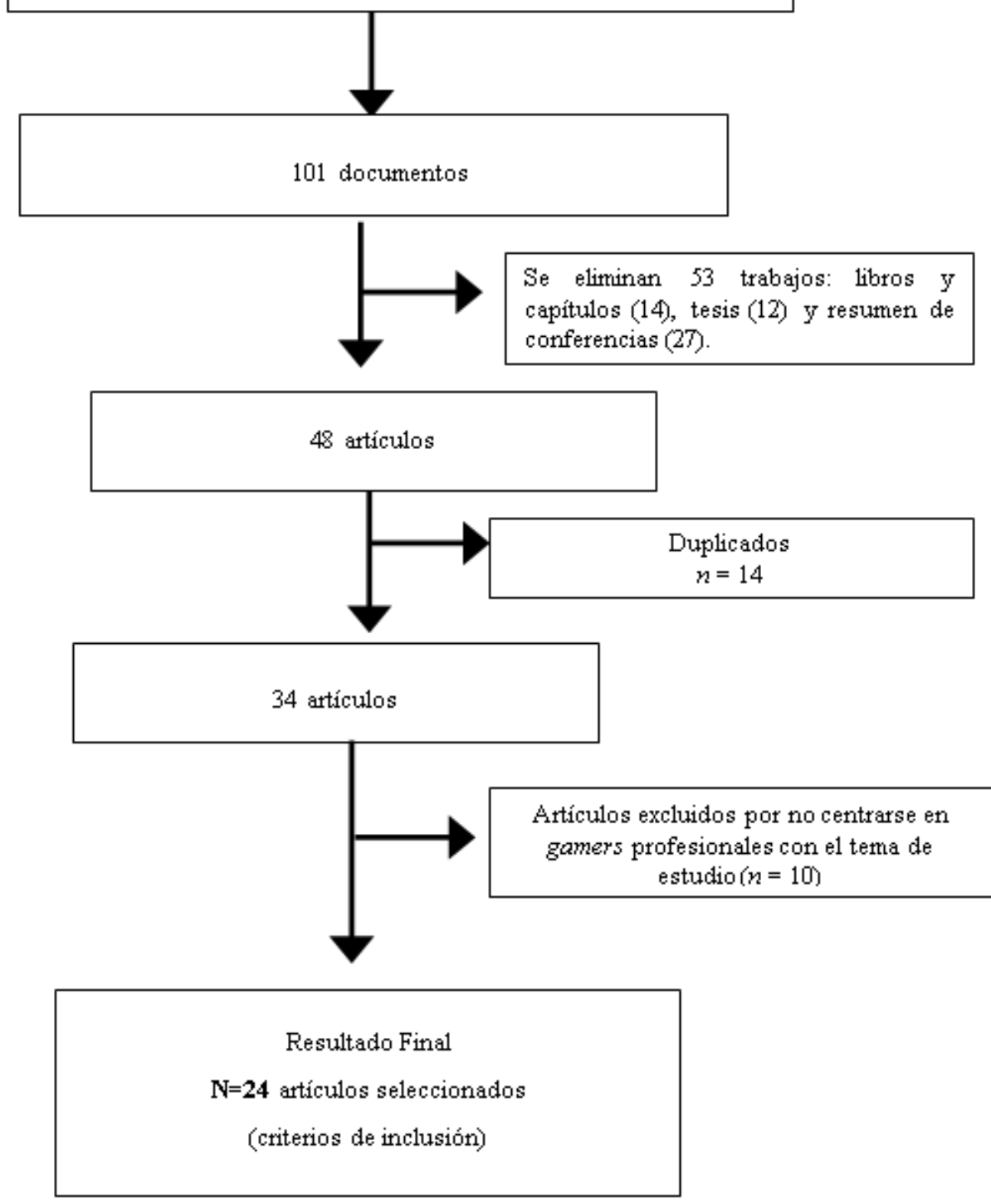

Figura 1. Diagrama de flujo PRISMA de Psicología y gamers profesionales en esports (a partir de Urrutia \& Bonfill, 2010).

\section{Síntesis de investigación}

El análisis temático es un método para identificar, analizar e informar patrones (temas) dentro de los datos y no suscribe ningún compromiso teórico implícito (Braun \& Clarke, 2006). En esta investigación, se ha utilizado el análisis temático para encontrar, a través de los artículos revisados, patrones repetidos de significado. Se ha explorado la frecuencia de cada tema de orden superior para establecer la popularidad del contenido en la investigación sobre Psicología y gamers profesionales en esports. 
El propósito de esta propuesta es establecer componentes de estudio del gamer profesional, que se han explorado con mayor o menor frecuencia en el contexto de la Psicología y los esports, pero también para ayudar a identificar temáticas comunes y las que no lo son. Desde este punto, establecer una agenda de investigación futura desde una perspectiva científica-profesional.

\section{Resultados}

Se revisaron un total de 101 artículos, de los que se seleccionaron 24 trabajos para la revisión y análisis según los criterios de inclusión marcados. Los estudios valorados giran en torno a 8 temáticas: entrenamiento psicológico, características de personalidad, procesos de equipo, consecuencias negativas de los esports, carrera professional, beneficios de los esports, habilidades y procesos psicológicos y entrenamiento invisible. Además, se clasificaron, según su tipología, en investigaciones $(n=16)$, revisiones $(n=4)$ y experiencias profesionales del psicólogo $(n=4)$.Ver Tabla 1 Tabla 1.

Resumen de artículos revisados $(n=24)$, tipo de estudios, tema establecido y título de trabajo sobre Psicología y gamers profesionales en esports entre los años 2012 y 13 de junio de 2020.

\begin{tabular}{|c|c|c|c|}
\hline Autores & Tipo de estudio & Tema & Título del trabajo \\
\hline $\begin{array}{l}\text { Poulus et al. } \\
(2020)\end{array}$ & Investigación & $\begin{array}{l}\text {-Habilidades y } \\
\text { procesos } \\
\text { psicológicos } \\
\text {-Entrenamiento } \\
\text { psicológico }\end{array}$ & $\begin{array}{l}\text { Stress and coping in esports and the } \\
\text { influence of mental toughness }\end{array}$ \\
\hline $\begin{array}{l}\text { Pedraza- } \\
\text { Ramirez et al. } \\
(2020)\end{array}$ & Revisión & $\begin{array}{l}\text {-Beneficios de los } \\
\text { esports }\end{array}$ & $\begin{array}{l}\text { Setting the scientific stage for esports } \\
\text { psychology: a systematic review }\end{array}$ \\
\hline $\begin{array}{l}\text { Macedo y } \\
\text { Falcão (2020) }\end{array}$ & Investigación & $\begin{array}{l}\text {-Procesos de } \\
\text { equipo. }\end{array}$ & $\begin{array}{l}\text { Like a pro: communication, } \\
\text { camaraderie and group cohesion in the } \\
\text { amazonian esports scenario }\end{array}$ \\
\hline $\begin{array}{l}\text { Maciej et al. } \\
(2020)\end{array}$ & Investigación & $\begin{array}{l}\text {-Habilidades y } \\
\text { procesos } \\
\text { psicológicos }\end{array}$ & $\begin{array}{l}\text { Social challenge and threat predict } \\
\text { performance and cardiovascular } \\
\text { responses during competitive video } \\
\text { gaming }\end{array}$ \\
\hline $\begin{array}{l}\text { Smith et al. } \\
\text { (2019) }\end{array}$ & Investigación & $\begin{array}{l}\text {-Habilidades y } \\
\text { procesos } \\
\text { psicológicos } \\
\text {-Entrenamiento } \\
\text { psicológico }\end{array}$ & $\begin{array}{l}\text { Identifying stressors and coping } \\
\text { strategies of elite esports competitors }\end{array}$ \\
\hline $\begin{array}{l}\text { Bányai et al. } \\
\text { (2019) }\end{array}$ & Investigación & $\begin{array}{l}\text {-Características de } \\
\text { personalidad. } \\
\text {-Consecuencias } \\
\text { negativas de los } \\
\text { esports }\end{array}$ & $\begin{array}{l}\text { The mediating effect of motivations } \\
\text { between psychiatric distress and } \\
\text { gaming disorder among esport gamers } \\
\text { and recreational gamers }\end{array}$ \\
\hline
\end{tabular}




\begin{tabular}{|c|c|c|c|}
\hline $\begin{array}{l}\text { Bonnar et al. } \\
\text { (2019) }\end{array}$ & Revisión & $\begin{array}{l}\text {-Entrenamiento } \\
\text { invisible }\end{array}$ & $\begin{array}{l}\text { Sleep and performance in Eathletes: for } \\
\text { the win! }\end{array}$ \\
\hline $\begin{array}{l}\text { Mora-Cantallops } \\
\text { y Sicilia (2019) }\end{array}$ & Investigación & $\begin{array}{l}\text {-Procesos de } \\
\text { equipo }\end{array}$ & $\begin{array}{l}\text { Team efficiency and network structure: } \\
\text { The case of professional league of } \\
\text { legends }\end{array}$ \\
\hline $\begin{array}{l}\text { Toth et al. } \\
\text { (2019) }\end{array}$ & Investigación & $\begin{array}{l}\text {-Habilidades y } \\
\text { procesos } \\
\text { psicológicos }\end{array}$ & $\begin{array}{l}\text { The color-word stroop task does not } \\
\text { differentiate cognitive inhibition ability } \\
\text { among esports gamers of varying } \\
\text { expertise }\end{array}$ \\
\hline $\begin{array}{l}\text { Freeman y } \\
\text { Wohn (2019) }\end{array}$ & Investigación & $\begin{array}{l}\text {-Procesos de } \\
\text { equipo. }\end{array}$ & $\begin{array}{l}\text { Understanding eSports team formation } \\
\text { and coordination }\end{array}$ \\
\hline $\begin{array}{l}\text { García-Naveira } \\
(2019)\end{array}$ & $\begin{array}{l}\text { Experiencia } \\
\text { profesional del } \\
\text { psicólogo }\end{array}$ & $\begin{array}{l}\text {-Entrenamiento } \\
\text { psicológico }\end{array}$ & $\begin{array}{l}\text { MAD Lions Esports Club: Experiencia } \\
\text { profesional del psicólogo del deporte }\end{array}$ \\
\hline Herrera (2019) & $\begin{array}{l}\text { Experiencia } \\
\text { profesional del } \\
\text { psicólogo }\end{array}$ & $\begin{array}{l}\text {-Entrenamiento } \\
\text { psicológico }\end{array}$ & $\begin{array}{l}\text { Integración de una psicóloga del deporte } \\
\text { en un club de eSports explicado a través } \\
\text { del modelo IPOD }\end{array}$ \\
\hline Mendoza (2019) & $\begin{array}{l}\text { Experiencia } \\
\text { profesional del } \\
\text { psicólogo }\end{array}$ & $\begin{array}{l}\text {-Entrenamiento } \\
\text { psicológico }\end{array}$ & $\begin{array}{l}\text { El trabajo psicológico online con } \\
\text { equipos de esports: experiencias en } \\
\text { Vodafone Giants }\end{array}$ \\
\hline $\begin{array}{l}\text { Pedraza- } \\
\text { Ramirez (2019) }\end{array}$ & $\begin{array}{l}\text { Experiencia } \\
\text { profesional del } \\
\text { psicólogo }\end{array}$ & $\begin{array}{l}\text {-Entrenamiento } \\
\text { psicológico }\end{array}$ & $\begin{array}{l}\text { Generación LoL: entrenamiento } \\
\text { psicológico mediante una propuesta } \\
\text { holística con un equipo profesional de } \\
\text { esports }\end{array}$ \\
\hline $\begin{array}{l}\text { García y } \\
\text { Chamorro } \\
(2018)\end{array}$ & Investigación & $\begin{array}{l}\text {-Características de } \\
\text { personalidad } \\
\text {-Entrenamiento } \\
\text { psicológico } \\
\text {-Entrenamiento } \\
\text { invisible }\end{array}$ & $\begin{array}{l}\text { Basic psychological needs, passion and } \\
\text { motivations in amateur and semi- } \\
\text { professional eSports players }\end{array}$ \\
\hline $\begin{array}{l}\text { Parshakov et al. } \\
\text { (2018) }\end{array}$ & Investigación & $\begin{array}{l}\text {-Procesos de } \\
\text { equipo }\end{array}$ & $\begin{array}{l}\text { Is diversity good or bad? Evidence from } \\
\text { eSports teams analysis. }\end{array}$ \\
\hline $\begin{array}{l}\text { Bányai et al. } \\
\text { (2018) }\end{array}$ & Revisión & $\begin{array}{l}\text {-Carrera } \\
\text { professional. } \\
\text {-Consecuencias } \\
\text { negativas de los } \\
\text { esports }\end{array}$ & $\begin{array}{l}\text { The psychology of esports: a systematic } \\
\text { literature review }\end{array}$ \\
\hline $\begin{array}{l}\text { Choi et al. } \\
\text { (2018) }\end{array}$ & Investigación & $\begin{array}{l}\text {-Consecuencias } \\
\text { negativas de los } \\
\text { esports }\end{array}$ & $\begin{array}{l}\text { Impact of the family environment on } \\
\text { juvenile mental health: eSports online } \\
\text { game addiction and delinquency }\end{array}$ \\
\hline $\begin{array}{l}\text { García-Naveira } \\
\text { et al. }(2018)\end{array}$ & Revisión & $\begin{array}{l}\text {-Beneficios de los } \\
\text { esports }\end{array}$ & $\begin{array}{l}\text { Beneficios cognitivos, psicológicos y } \\
\text { personales del uso de los videojuegos y } \\
\text { esports: una revisión }\end{array}$ \\
\hline $\begin{array}{l}\text { Pérez-Rubio et } \\
\text { al. (2017) }\end{array}$ & Investigación & $\begin{array}{l}\text {-Características de } \\
\text { personalidad. }\end{array}$ & $\begin{array}{l}\text { Personalidad y burnout en jugadores } \\
\text { profesionales de e-sports }\end{array}$ \\
\hline $\begin{array}{l}\text { Himmelstein et } \\
\text { al. (2017) }\end{array}$ & Investigación & $\begin{array}{l}\text {-Habilidades y } \\
\text { procesos } \\
\text { psicológicos } \\
\text {-Entrenamiento } \\
\text { psicológico }\end{array}$ & $\begin{array}{l}\text { An exploration of mental skills among } \\
\text { competitive league of legend players }\end{array}$ \\
\hline Seo (2016) & Investigación & $\begin{array}{l}\text {-Carrera } \\
\text { professional. }\end{array}$ & $\begin{array}{l}\text { Professionalized consumption and } \\
\text { identity transformations in the field of } \\
\text { eSports }\end{array}$ \\
\hline $\begin{array}{l}\text { Martončik } \\
(2015)\end{array}$ & Investigación & $\begin{array}{l}\text {-Características de } \\
\text { personalidad. }\end{array}$ & $\begin{array}{l}\text { e-Sports: Playing just for fun or playing } \\
\text { to satisfy life goals?. }\end{array}$ \\
\hline $\begin{array}{l}\text { Han et al. } \\
(2012)\end{array}$ & Investigación & $\begin{array}{l}\text {-Consecuencias } \\
\text { negativas de los } \\
\text { esports }\end{array}$ & $\begin{array}{l}\text { Differential regional gray matter } \\
\text { volumes in patients with on-line game } \\
\text { addiction and professional gamers }\end{array}$ \\
\hline
\end{tabular}


A continuación, se presentan los 8 temas establecidos según su frecuencia de aparición y breve descripción del contenido que le define. Ver Figura 2.

Tema 1: Entrenamiento psicológico ( $n=8$ artículos). Hace referencia a los trabajos que citan la necesidad de la intervención psicológica o experiencia profesional del psicólogo/a con los gamers, por ejemplo, para el crecimiento personal y/o mejora del rendimiento en los esports (García \& Chamorro, 2018; García-Naveira, 2019; Herrera, 2019; Himmelstein et al., 2017; Mendoza, 2019; Pedraza-Ramirez, 2019; Poulus et al., 2020; Smith et al., 2019).

Tema 2. Habilidades y procesos psicológicos ( $n=5$ artículos). Hace referencia a los trabajos que buscan identificar factores psicológicos que intervienen en el rendimiento de los gamers, por ejemplo, habilidades de afrontamiento al estrés, obstáculos detectados y procesos psicológicos implicados (Himmelstein et al., 2017; Maciej et al., 2020; Poulus et al., 2020; Smith et al., 2019; Toth et al., 2019).

Tema 3: Características de personalidad ( $n=4$ artículos). Hace referencia a los trabajos que describen cómo son los gamers, por ejemplo, analizando los rasgos de personalidad y motivaciones (Bányai et al., 2019; García \& Chamarro, 2018; Martončik, 2015; Pérez-Rubio et al., 2017).

Tema 4: Procesos de equipo ( $n=4$ artículo). Hace referencia a los trabajos que abordan las variables implicadas en los equipos, por ejemplo, la constitución y desarrollo del equipo para obtener un mayor rendimiento en esports (Freeman \& Wohn, 2019; Macedo \& Falcão, 2020; Mora-Cantallops \& Sicilia, 2019; Parshakov et al., 2018).

Tema 5. Consecuencias negativas de los esports $(n=4$ artículos). Hace referencia a los trabajos que indican el posible efecto negativo de una dedicación excesiva del videojuego y otros factores asociados sobre la salud de los gamers (Bányai et al., 2018; Bányai et al., 2019; Choi et al., 2018; Han et al., 2012).

Tema 6: Carrera professional ( $n=2$ artículos), Hace referencia a los trabajos que indican el proceso por el cual pasa una persona hasta llegar a ser un gamer professional (Bányai et al., 2018; Seo, 2016).

Tema 7: Beneficios de los esports $(n=2$ artículos). Hace referencia a los trabajos que recogen las aportaciones cognitivas, psicológicas y personales de los esports sobre el desarrollo y rendimiento de los gamers (García-Naveira et al., 2018; Pedraza-Ramirez et al., 2020).

Tema 8. Entrenamiento invisible ( $n=2$ artículos). Hace referencia a los trabajos que destacan los aspectos externos del videojuego (out game) que afectan a la vida y el 
rendimiento del gamer, como, por ejemplo, la alimentación y el descanso (Bonnar et al., 2019; García \& Chamarro, 2018).

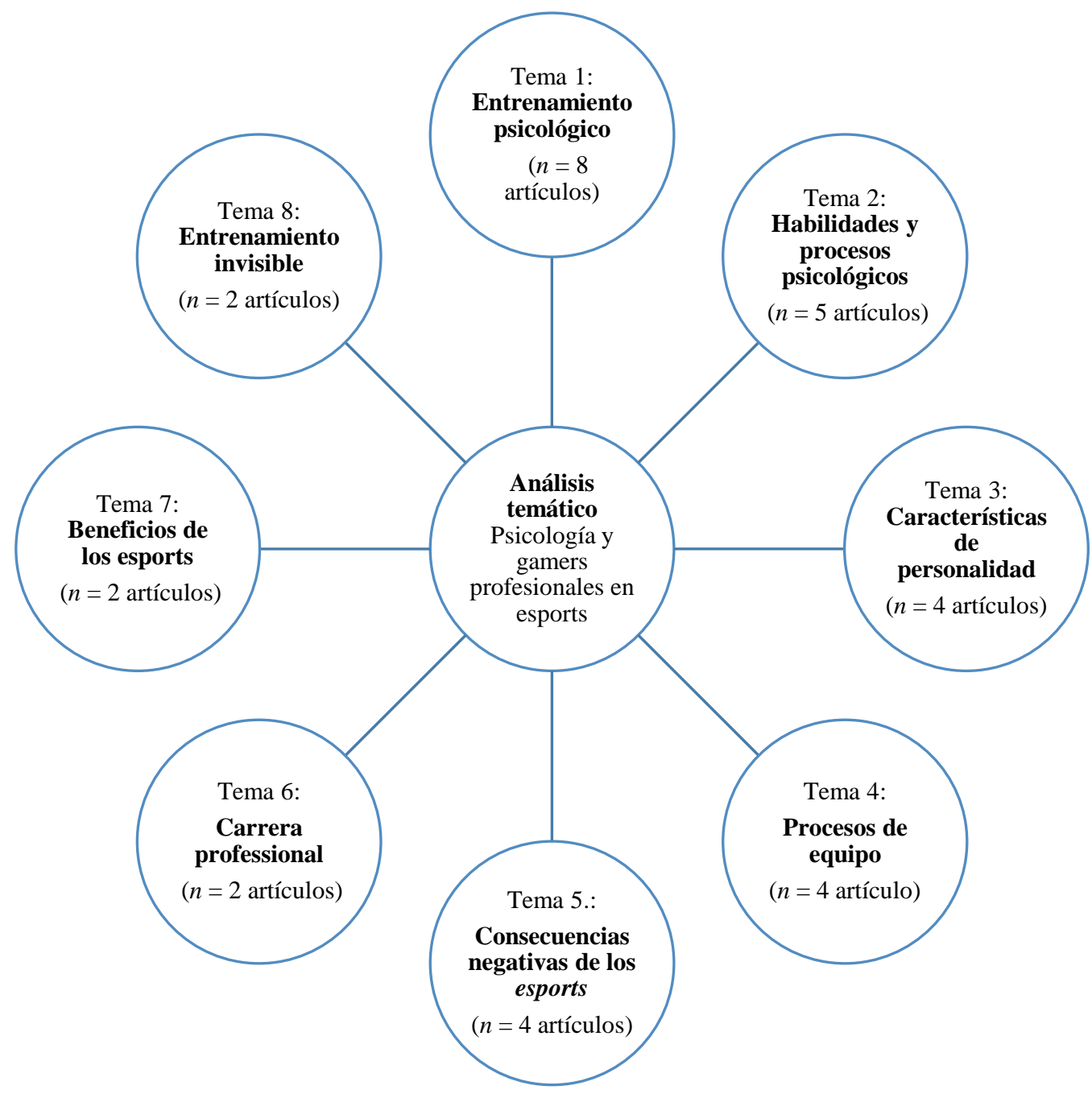

Figura 2. Análisis temático sobre Psicología y gamers profesionales en esports (2012-13 de junio de 2020).

Respecto a la relación entre los años de publicación revisados (desde el 2012 hasta el 13 de junio de 2020) y la producción científica-profesional sobre la temática $(n=24)$, al establecer períodos de 3 años, entre los años 2012-2014 se publicó 1 trabajo (4 \% del total), 2015-2017 se publicaron 4 trabajos (17\% del total) y 2018-13 de junio de 2020 se publicaron 19 trabajos (79\% del total). Ver Figura 3. 


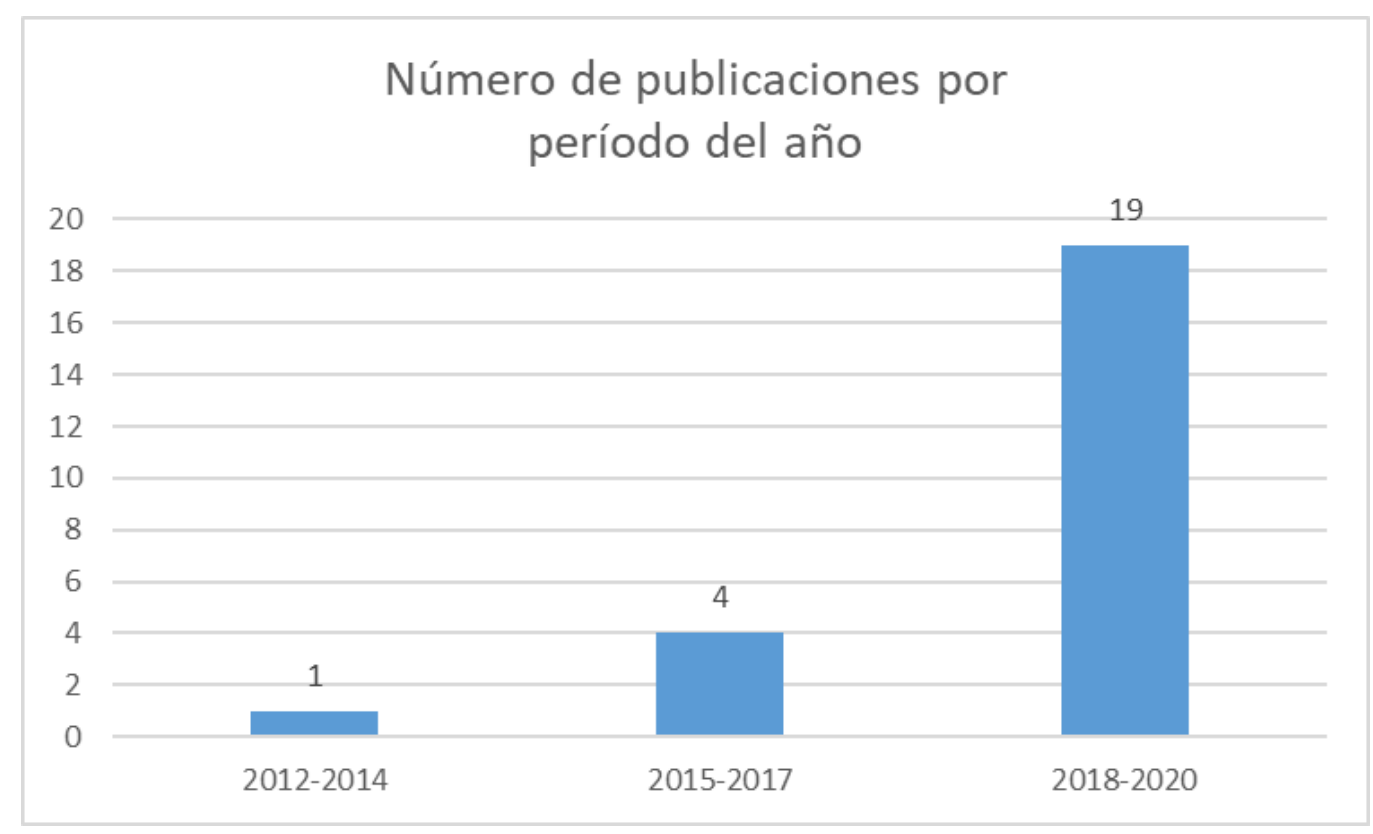

Figura 3. Número de trabajos revisados sobre Psicología y gamer profesional en esports $(n=24)$ en función del período de publicación (2012-2014, 2015-2017 y 2018-13 de junio de 2020)

En relación a la distribución de artículos publicados en función de la revista, es importante señalar que 5 fueron en la Revista de Psicología Aplicada al Deporte y al Ejercicio Físico y 2 en Frontiers in Psychology, mientras que el resto de artículos fueron publicados en diferentes revistas, lo que imposibilita su agrupación en categorías.

\section{Discusión}

Las revisiones bibliográficas para el análisis temático se han propuesto como un método útil para identificar brechas potenciales en un cuerpo de investigación y proporcionar una base para una decisión informada sobre si llevar a cabo una investigación o trabajo aplicado. Este estudio tuvo como objetivo desarrollar un análisis temático de artículos revisados sobre Psicología y gamers profesionales en esports. En total, 24 artículos seleccionados se clasificaron en 8 temas basados en su análisis de contenido. Los temas más tratados son el entrenamiento psicológico ( $n=8$ artículos) y las habilidades y procesos psicológicos ( $n=5$ artículos), lo que representa una tendencia al interés por la preparación y competencias mentales del gamer profesional asociados a la mejora del rendimiento en los esports.

Los datos tienden a indicar que la producción de trabajos sobre la temática ha sido algo más baja $(n=24)$ si lo comparamos con las revisiones de Pedraza-Ramirez et al. (2020) con 52 trabajos, Bányai et al. (2018) con 30 trabajos y García-Naveira et al. (2018) con 26 trabajos, los cuales han abordado de manera más global el sector desde la 
Psicología y los esports, y no específicamente sobre la población de gamers profesionales como en el presente trabajo, en el que se percibe la necesidad de ampliar y profundizar sobre los temas tratados, que se presentará en el apartado de agenda de investigación. Estos resultados van en la línea de otros trabajos en los que se concluye la necesidad de avanzar en el estudio psicológico del gamer profesional en esports, atendiéndolo como una actividad y población específica, diferente al uso de los videojuegos y el gamer ocasional (Bányai et al., 2019; Bányai et al., 2018; Pérez-Rubio et al., 2017),

Además, se observa una tendencia al alza en cuanto a períodos de producción científica-profesional, en que los años 2018- 13 de junio de 2020 ( $n=19$ artículos) registró un mayor número que en los años 2015-2017 $(n=4)$ y 2012-2015 $(n=1)$, posiblemente en paralelo al crecimiento del sector de los esports, aunque, como indican otros autores (Hulk, 2019; Martin-Niedecken \& Schättin, 2020; Pluss et al., 2019), el tema sigue siendo relativamente nuevo para los investigadores y profesionales.

Respecto a la tipología de los artículos que se incluyeron en este estudio, el análisis desprende que son de investigación mayoritariamente $(n=16)$, aunque se han realizado tanto de revisión $(n=4)$ como trabajos de experiencia profesional del psicólogo/a $(n=4)$ en los esports. Este trinomio de publicaciones (investigación-revisiónexperiencia) puede ser una base fructífera para el desarrollo científico-profesional en los esports. En este caso, la revista que publicó un mayor número de artículos, es una revista española, la Revista de Psicología Aplicada al Deporte y al Ejercicio Físico $(n=5)$, cuyos artículos son de corte de experiencia profesional.

Además, del objetivo principal que surgen del análisis temático, hay una serie de direcciones más específicas que la investigación futura podría tomar para avanzar en la comprensión del gamer profesional desde la Psicología. Brevemente, se describirá áreas potenciales de trabajo $(n=10)$ que pueden mejorar la comprensión del papel de la Psicología en los esports en general, y en los gamers profesionales en particular.

\section{Agenda de investigación}

1. El psicólogo/a como profesión imprescindible en los esports. Los esports al tener un alto contenido mental, requiere que los clubes contraten a los psicólogos/as dentro de su organigrama de trabajo, y desde este posicionamiento, se necesitan más trabajos de experiencia profesional que traten cuestiones como las establecidas en el presente trabajo. 
Otra cuestión relevante a definir es el perfil del psicólogo/a que trabaja en esports, ya que, actualmente, no hay un marco legal de referencia, acreditación profesional o itinerario formativo establecido. Tal y como como indican García-Naveira y Cantón (2020) la psicología del deporte y su ejercicio profesional tienen un grado de delimitación y consistencia suficiente como para integrar la labor con los esports, convirtiéndose en un nuevo sub-ámbito emergente de intervención del psicólogo/a del deporte, tras una formación y experiencia complementaria para considerarse como experto/a en esports.

2. Procesos psicológicos implicados en el rendimiento. El conocimiento general de cómo funciona la mente del gamer, sus procesos motivacionales y atencionales, cómo afronta la competición, qué obstáculos lo limitan y qué habilidades de afrontamiento podría utilizar para superarlo, son retos a profundizar en la investigación futura, debido a que, aunque existe un cierto paralelismo con el deporte, puede diferir el perfil del gamer vs. deportista y demandas de los esports vs. deporte.

Además, desde una perspectiva profesional, se requiere entrenar a los gamers en habilidades psicológicas de autorregulación emocional, ya que se percibe que padecen estrés y baja tolerancia a la frustración, ya sea por la exigencia de la competición, la alta dedicación al videojuego, falta de preparación integral y hábitos saludables y/o baja fortaleza mental. También, el entrenamiento en valores (liderazgo, compromiso, esfuerzo, trabajo, disciplina y superación), complementaría al crecimiento personal y deportivo del gamer, debido a que es posible que exista una carencia en esta cuestión a causa de falta de experiencias previas en contextos que los potencian desde edades tempranas, tal y como sucede en el deporte.

3. Estudio de las diferencias individuales. El conocimiento del perfil del gamer podría integrar el estudio de características de personalidad (e.g., rasgos y motivaciones) en función de la edad y sexo, así como compararlos con otras poblaciones (p.ej., gamer ocasional, deportistas y población general).

Por ejemplo, en relación al punto 2, los rasgos de estabilidad emocional y responsabilidad se asocian con un mayor rendimiento deportivo (García-Naveira y RuizBarquín, 2020), cuestión que se podría estudiar entre los gamers, y a partir de estos datos, desarrollar programas de intervención psicosociales para la mejora del rendimiento y el cuidado de la salud.

4. Selección y desarrollo de equipos de alto rendimiento. En primer lugar, se podría optimizar el proceso de selección de los gamers a los equipos, ya que habitualmente se realiza de forma subjetiva y poco rigurosa (por amistad, lo que se conoce 
del jugador, etc.). Para ello, se podría seguir los modelos que se utilizan en el ámbito empresarial y deportivo (definir el perfil y competencias del puesto de trabajo a cubrir, evaluar y seleccionar), con la intervención de diferentes profesionales (director deportivo, jugadores, preparador físico y psicólogo/a) y distintas metodologías de evaluación (entrevista, batería de test, pruebas situacionales o período de prueba, etc.). En segundo lugar, se podría potenciar los equipos, ya que muchos de los gamers no saben qué es estar y rendir en equipo. Por ejemplo, los niños/as comienzan a edades tempranas (e.g., 5 años) a jugar al fútbol, aprendiendo lo que es estar en un entorno social, relacionarse con los demás, el trabajar en equipo, respetar las normas, el rol del entrenador, etc., cuestión que no queda bien definida en jóvenes que no hacen o han hecho deporte, pudiendo llegar a ser una debilidad a atender. Por ello es importante desarrollar al equipo en cuanto a su estructura y funcionamiento (e.g., establecimiento de roles, estatus, normas, procesos y valores), así como el entrenamiento psicológico en variables de equipo como el trabajo en equipo, la cohesión, coordinación y cooperación.

5. Prevención y atención psicológica. Se requiere realizar investigaciones y trabajos de experiencias profesionales interdisciplinares (e.g., psicólogos/as y médicos) sobre programas preventivos, promoción de la salud y de atención psicológica de los gamers, especialmente en adolescentes. Se ha observado que suele haber un uso excesivo de los videojuegos y falta de hábitos saludables que producen una vulnerabilidad psicológica a ciertas psicopatologías (ansiedad, estrés, depresión y burnout). Por un lado, es necesario educar y formar a los gamers en estas cuestiones, y por otro, garantizar que las personas que practican esports y desarrollan problemas, obtengan ayuda, apoyo y tratamiento si lo necesitan.

Otras cuestiones a atender son la necesidad de delimitar cuántas horas de entrenamiento son necesarias para un alto rendimiento saludable en los esports y cuántas son malas, así como definir si los gamers que experimentan problemas psicológicos graves asociados a los esports serían clasificados como adictos al juego o al trabajo.

6. Desarrollo de programas de carreras profesionales. Sería interesante llevar a cabo programas de detección y desarrollo de talentos en esports, en el que exista un proyecto del gamers, detectando fortalezas, áreas de mejora y dificultades a las que se puede encontrar. Otro tema es el entrenamiento integral para potenciar el talento (físico, técnico-táctico y psicológico) y la salud de los gamers, así como la promoción de carreras duales (compaginar esports y estudios), cuestión bastante asentada en el deporte y que puede servir de referencia. 
Además, estudiar si actualmente se sigue el mismo proceso de carrera del gamer, ya que antiguamente se hacía partiendo del ocio y jugando desde casa hasta llegar a ser fichado por un club, o si existe una vía alternativa para los jóvenes que quieren llegar al profesionalismo y buscar caminos alternativos (e.g., academias de esports, equipos filiales de clubes profesionales o torneos de aficionados), por lo que el escenario pudiera ser distinto, al igual que los tiempos (o prisas) y dificultades del proceso, así como el impacto en la persona y su entorno. Por último, este tema también plantea una pregunta teórica interesante, si algunos gamers ven su actividad profesional como su trabajo o como una actividad de ocio, así como qué sucede con ellos cuando se retiran (alrededor de los 25 años, por ejemplo, por burnout).

7. Estudio positivo de los esports. El uso responsable de los esports en cuanto al número de horas de uso, sumados a unos buenos hábitos saludables (actividad física, descanso y alimentación), el compaginarlos con otras actividades (e.g., estudios o trabajo) y el tener relaciones sociales (presenciales y online), pueden ser una excelente base para beneficiarse de los efectos positivos de los esports. Para ello, se requieren más trabajos que aborden esta perspectiva y que complementen a los estudios que se centran en los efectos negativos que pueden llegar a producir.

También hay que descubrir si todos los esports cumplen la misma función y tienen las mismas necesidades (cognitivas y psicológicas), en que las herramientas de imagen y psicofisiológicas facilitarán esta comprensión como posibles predictores de rendimiento entre los gamers debido a la validez ecológica que los esports pueden proporcionar. Po su parte, los psicólogos/as se beneficiarán al comprender los mecanismos cognitivos y habilidades psicológicas subyacentes de cada esport, para adaptar las estrategias de entrenamiento a las especificidades de cada videojuego.

8. Entrenamiento invisible, bienestar y rendimiento. Se requiere medir el impacto sobre el gamer de los aspectos que integran el entrenamiento invisible (e.g., actividad física, descanso y alimentación), así como el desarrollo de programas que potencien estas cuestiones y beneficien la salud y el rendimiento de la persona. Este es un asunto importante a estudiar e intervenir, ya que una alta dedicación a los esports puede tener un impacto negativo sobre los hábitos saludables y la salud de los gamers (sedentarismo, insomnio alimentación y mala alimentación). Por tanto, se requiere instaurar una cultura de alto rendimiento que integre y cuide tanto todos los aspectos relacionados entrenamientos "in game" como lo relacionado con el entrenamiento "out game", aspecto que está bastante integrado en el deporte como referente. 
9. Investigaciones desde la neuropsicología. El conocimiento de las estructuras y funciones del cerebro, así como la influencia de los esports (e.g., definiendo tipo de videojuego y horas de dedicación) sobre ellas, podría abrir una venta al entrenamiento cerebral y la salud del gamer, por lo que se requieren estudios hacia esta dirección. También analizar posibles diferencias respecto a otras poblaciones (e.g., gamer ocasional, deportistas y población en general).

10. Otros factores psicológicos $\mathbf{y}$ entrenadores. Existen diferentes temas estudiados en Psicología del Deporte con deportistas que pueden servir de guía en el estudio de los gamers (resiliencia, ansiedad-estrés, liderazgo, etc.), así como otros colectivos, como son los entrenadores de los esports, en el que no se ha observado ningún estudio específico y son un referente importante de gestión personal y rendimiento de los gamers. Además, este colectivo cobra un especial interés, ya que actualmente no existe una formación reglada y oficial de entrenador de esports, los cuales suelen ser ex gamers o analistas del juego.

\section{Limitaciones}

Esta revisión bibliográfica de 24 artículos, con 8 temas relacionados y 10 áreas potenciales de trabajo en Psicología y gamers profesionales en esports, tiene una serie de limitaciones potenciales que requieren consideración. Primero, el análisis de referencias se limita al alcance de las bases de datos incluidas. Es probable que algunos artículos se hayan perdido si se publican predominantemente otras bases de datos. Sin embargo, las 3 bases de datos elegidas tienen una amplitud considerable y es probable que la mayoría de los artículos hayan aparecido en esas bases de datos. En otras palabras, la evidencia se limita al alcance de las bases de datos elegidas y no necesariamente refleja la investigación basada en todo el universo de trabajos publicados en su conjunto. 


\section{Referencias}

Antón, M. (2019). Los deportes electrónicos (esports): el espectáculo de las competiciones de videojuegos. [Tesis doctoral, Universidad Complutense de Madrid]. https://bit.ly/2Y8G2J7

Bányai, F., Griffiths, M., Demetrovics, Z., \& Király, O. (2019). The mediating effect of motivations between psychiatric distress and gaming disorder among esport gamers and recreational gamersç. Comprehensive Psychiatry, 94. https://doi.org/10.1016/j.comppsych.2019.152117

Bányai, F., Griffiths, M., Király, O., \& Demetrovics, Z. (2018). The psychology of esports: a systematic literature review. Journal of Gambling Studies, 35, 351365. https://doi.org/10.1007/s10899-018-9763-1

Braun, V., \& Clarke, V. (2006). Using thematic analysis in psychology. Qualitative Research in Psychology, 3(2), 77-101. https://doi.org/10.1191/1478088706qp063oa

Bonnar, D., Castine, B., Kakoschke, N., \& Sharp, G. (2019). Sleep and performance in Eathletes: for the win!. Sleep Health, 5(6), 647-650. https://doi.org/10.1016/j.sleh.2019.06.007

Campbell, M. J., Toth, A. J., Moran, A. P., Kowal, M., \& Exton, C. (2018). eSports: A new window on neurocognitive expertise? Progress in brain research, 240, 161-174. https://doi.org/10.1016/bs.pbr.2018.09.006

Chiva-Bartoll, O., Pallarès-Piquer, M., \& Isidori, E. (2018). Esports y deportes convencionales: cuestiones éticas y pedagógicas derivadas de la participación corporal. Cultura, Ciencia y Deporte, 40(14), 71-79. http://dx.doi.org/10.12800/ccd.v14i40.1227

Choi, C., Hums, M., \& Bum, C. H. (2018). Impact of the family environment on juvenile mental health: eSports online game addiction and delinquency. International Journal of Environmental Research and Public Health, 15(12), 2850. http://dx.doi.org/10.3390/ijerph15122850

DEV (2018). Libro blanco del desarrollo español de videojuegos. https://bit.ly/2zztFeY

Freeman, G. \& Wohn, D. Y. (2019). Understanding eSports team formation and coordination. Computer supported cooperative work (CSCW), 28(1-2), 95126. https://doi.org/10.1007/s10606-017-9299-4

García, S. \& Chamorro, A. (2018). Basic psychological needs, passion and motivations in amateur and semi-professional eSports players. Revista de Psicologia, Ciències de l'Eduació i de l'Esport, 36(2), 59-68.

García-Naveira, A. (2019). MAD Lions Esports Club: Experiencia profesional del psicólogo del deporte. Revista de Psicología Aplicada al Deporte y al Ejercicio Físico, 4, e7. https://doi.org/10.5093/rpadef2019a6

García-Naveira, A. y Cantón, E. (2020). Perfil profesional del psicólogo/a del deporte experto/a en esports. Revista de Psicología Aplicada al Deporte y al Ejercicio Físico, 5, e13. https://doi.org/10.5093/rpadef2020a9

García-Naveira, A. y Ruiz-Barquín, R. (2020). Personalidad y rendimiento deportivo en jugadores de fútbol desde el modelo de Millón. Anuario de Psicología, 50(3), 135-148.

García-Naveira, A., Jiménez, M., Teruel, B., \& Suárez, A. (2018). Beneficios cognitivos, psicológicos y personales del uso de los videojuegos y esports: una revisión. Revista de Psicología Aplicada al Deporte y al Ejercicio Físico, 3, e16. https://doi.org/105093/rpadef2018a15 
Grant, M. J. \& Booth, A. (2009). A typology of reviews: An analysis of 14 review types and associated methodologies. Health Information \& Libraries Journal, 26(2), 91-108. https://doi.org/10.1111/j.1471-1842.2009.00848.x

Hamari, J. \& Sjöblom, M. (2017). What is eSports and why do people watch it? Internet Research, 27(2), 211-232. https://doi.org/10.1108/IntR-04-2016$\underline{0085}$

Han, D. H., Lyoo, I. K., \& Renshaw, P. F. (2012). Differential regional gray matter volumes in patients with on-line game addiction and professional gamers. Journal of psychiatric research, 46(4), 507-515. https://doi.org/10.1016/j.jpsychires.2012.01.004

Herrera. M (2019). Integración de una psicóloga del deporte en un club de eSports explicado a través del modelo IPOD. Revista de Psicología Aplicada al Deporte y al Ejercicio Físico, 4, e3. https://doi.org/10.5093/rpadef2019a4

Himmelstein, D., Liu, Y., \& Shapiro, J. L. (2017). An exploration of mental skills among competitive league of legend players. International Journal of Gaming and Computer-Mediated Simulations,9(2),21. https://doi.org/10.4018/IJGCMS.2017040101

Hulk, T. (2019). The social context of the benefits achieved in esport. The New Educational Review, 55(1), 160-169. https://doi.org/10.15804/tner.2019.55.1.13

Jang, W. \& Byon, K. (2020). Antecedents of esports gameplay intention: genre as a moderator. Computers in Human Behavior, 109(106336). https://doi.org/10.1016/j.chb.2020.106336

Macedo, T. \& Falcão, T. (2020). Like a Pro: Communication, Camaraderie and Group Cohesion in the Amazonian Esports Scenario, Entertainment Computing, 34(100354). https://doi.org/10.1016/j.entcom.2020.100354

Maciej, B., Kosakowski, M., \& Kaczmarek, L. D. (2020). Social challenge and threat predict performance and cardiovascular responses during competitive video gaming. Psychology of Sport and Exercise, 46, 101584. https://doi.org/10.1016/j.psychsport.2019.101584

Martin-Niedecken' A. \& Schättin' A. (2020). Let the body'n'brain games begin: toward innovative training approaches in esports athletes. Frontiers in Psychology, 11(138). https://doi.org/10.3389/fpsyg.2020.00138

Martončik, M. (2015). e-Sports: Playing just for fun or playing to satisfy life goals?. Computers in Human Behavior, 48, 208-211. https://doi.org/10.1016/j.chb.2015.01.056

Mendoza, G. (2019). El trabajo psicológico online con equipos de esports: experiencias en Vodafone Giants. Revista de Psicología Aplicada al Deporte y al Ejercicio Físico, 4, e5. https://doi.org/10.5093/rpadef2019a5

Mora-Cantallops, M. \& Sicilia, M-Á. (2019). Team efficiency and network structure: The case of professional league of legends. Social Networks, 58, 105-115. https://doi.org/10.1016/j.socnet.2019.03.004

Newzoo (2019). Newzoo global esports market report 2019. Light version. https://bit.ly/3eR5hFX

ONTIER (2018). Guía legal sobre esports. Presente y futuro de la regulación de los esports en España. https://bit.ly/2KE56ja

Parry, J. (2018). E-sports are not sports. Sport, Ethics and Philosophy, 13(1), 3-18. https://doi.org/10.1080/17511321.2018.1489419 
Parshakov, P., Coates, D., \& Zavertiaeva, M. (2018). Is diversity good or bad? Evidence from eSports teams analysis. Journal Applied Economics 50(47), 5064-5075. https://doi.org/10.1080/00036846.2018.1470315

Pedraza-Ramirez, I. (2019). Generación LoL: entrenamiento psicológico mediante una propuesta holística con un equipo profesional de esports. Revista de Psicología Aplicada al Deporte y al Ejercicio Físico, 4, e2. https://doi.org/10.5093/rpadef2019a3

Pedraza-Ramirez, I., Musculus, L., Raab, M., \& Laborde, S. (2020). Setting the scientific stage for esports psychology: A systematic review, International Review of Sport and Exercise Psychology. https://doi.org/10.1080/1750984X.2020.1723122

Pérez-Rubio, C., González, J., \& Garcés de los Fayos, E.J. (2017). Personalidad y burnout en jugadores profesionales de e-sports. Cuadernos de Psicología del Deporte, 17(1), 41-50.

Pluss, M., Bennett, K. J. M., Novak, A. R., Panchuk, D., Coutts, A., \& Fransen, J. (2019). Esports: The Chess of the 21st Century. Frontiers in Psychology, 10, 156. https://doi.org/10.3389/fpsyg.2019.00156

Poulus' D., Coulter, T., Trotter, M., \& Polman, R. (2020). Stress and Coping in Esports and the Influence of Mental Toughness. Frontiers in Psychology, 11(628). https://doi.org/10.3389/fpsyg.2020.00628

Sánchez, A. \& Remilllard, J. (2018). Esport: towards a hermeneutic of virtual sport. Cultura, Ciencia y Deporte, 38(13), 137-145. http://dx.doi.org/10.12800/ccd.v13i38.1076

Seo, Y. (2016). Professionalized consumption and identity transformations in the field of eSports. Journal of Business Research, 69(1), 264-272. https://doi.org/10.1016/j.jbusres.2015.07.039

Smith, M. J., Birch, P. D., \& Bright, D. (2019). Identifying stressors and coping strategies of elite esports competitors. International Journal of Gaming and Computer-Mediated Simulations. 11, 22-39. https://doi.org/10.4018/ijgcms.2019040102

Stanton, R. (2015). A brief history of video games. Londres, Reino Unido: Robinson

Thiel, A. \& John, J. (2018). Is eSport a 'real' sport? Reflections on the spread of virtual competitions. European Journal for Sport and Society, 15(4), 311315. https://doi.org/10.1080/16138171.2018.1559019

Toth, A. J., Kowal, M., \& Campbell, M. J. (2019). The color-word stroop task does not differentiate cognitive inhibition ability among esports gamers of varying expertise. Frontiers in Psychology, 10(2852). http://dx.doi.org/10.3389/fpsyg.2019.02852

Urrutia G. \& Bonfill, X. (2010). Declaración PRISMA: una propuesta para mejorar la publicación de revisiones sistemáticas y metaanálisis. Medicina Clinica, 135(11),507-511. https://doi.org/10.1016/j.medcli.2010.0 